\title{
Successful Endoscopic Ultrasound-Guided Treatment of a Spontaneous Rupture of a Hemorrhagic Pancreatic Pseudocyst
}

\author{
Chan Park', Tae Hyeon Kim² and Hyung Ku Chon ${ }^{2}$ \\ Department of ${ }^{1}$ Surgery, ${ }^{2}$ Internal Medicine, Wonkwang University College of Medicine and Hospital, Iksan, Korea
}

Spontaneous rupture of a hemorrhagic pancreatic pseudocyst may be life-threatening. Endoscopic ultrasound (EUS)-guided drainage has been reported to be a valuable treatment option for pancreatic pseudocysts. However, its usefulness in the management of a ruptured pancreatic pseudocyst is limited. We report a rare case of a spontaneous rupture of a hemorrhagic pancreatic pseudocyst in a patient with chronic pancreatitis, which was successfully treated with EUS-guided gastrocystostomy with a fully covered self-expandable metallic stent. Clin Endosc 2021;54:763-766

Key Words: Endoscopic ultrasound; Gastrocystostomy; Pancreatic Pseudocyst

\section{INTRODUCTION}

Pancreatic pseudocyst is a well-known complication of acute and chronic pancreatitis. Most pseudocysts spontaneously regress with conservative treatment. ${ }^{1}$ However, serious complications, including cystic rupture, hemorrhage, infection, and pseudoaneurysm rupture, may increase the risk of morbidity and mortality. ${ }^{2}$ In particular, spontaneous rupture of a hemorrhagic pancreatic pseudocyst may be life-threatening. Herein, we report a rare case of a spontaneous rupture of a hemorrhagic pancreatic pseudocyst in a patient with chronic pancreatitis, which was successfully treated with endoscopic ultrasound (EUS)-guided gastrocystostomy with a fully covered self-expandable metallic stent (FCSEMS).

Received: October 21, 2020 Revised: December 23, 2020

Accepted: December 24, 2020

Correspondence: Hyung Ku Chon

Department of Internal Medicine, Wonkwang University College of Medicine and Hospital, 895 Muwang-ro, Iksan, Chonbuk 54538, Korea

Tel: +82-63-859-1676, Fax: +82-63-855-2025, E-mail: gipb2592@wku.ac.kr

ORCID: https://orcid.org/0000-0002-6068-3849

(c) This is an Open Access article distributed under the terms of the Creative Commons Attribution Non-Commercial License (http://creativecommons.org/ licenses/by-nc/3.0) which permits unrestricted non-commercial use, distribution, and reproduction in any medium, provided the original work is properly cited.

\section{CASE REPORT}

A 46-year-old man with alcoholic chronic pancreatitis was admitted to our hospital with severe upper abdominal pain and high fever (temperature up to $38.5^{\circ} \mathrm{C}$ ) for 1 day. A week ago, he was scheduled to undergo EUS-guided intervention for a pseudocyst in the pancreatic tail, about $9 \mathrm{~cm}$ in size, which was identified on magnetic resonance imaging (Fig. 1A). Laboratory tests revealed low hemoglobin $(9.8 \mathrm{~g} / \mathrm{dL})$ and elevated inflammatory markers (white blood cell count $17,500 / \mu \mathrm{L}$ and C-reactive protein $324.56 \mathrm{mg} / \mathrm{L}$ ). Abdominal computed tomography revealed that the hemorrhagic pancreatic pseudocyst had ruptured, with direct extension to the splenorenal ligament and subcapsular splenic space. However, there was no evidence of active contrast extravasation (Fig. 1B, C). EUS showed an anechoic thick-walled cystic lesion, $9 \mathrm{~cm}$ in size (Fig. 2A). Transgastric wall puncture was performed using a 19-gauge needle for fine-needle aspiration (EZ shot 3 plus ${ }^{\mathrm{TM}}$; Olympus, Tokyo, Japan), avoiding intervening vessels (Fig. 2B). Thick and turbid bloody fluid with debris was aspirated, and a 0.025-inch guidewire (Visiglide; Olympus Medical Systems, Tokyo, Japan) was coiled into the pseudocyst cavity under fluoroscopic guidance (Fig. 2C). Subsequently, tract dilation was performed using a 6-Fr cystotome (Wilson-Cook Medical Inc., Bloomington, IN, USA), and an FCSEMS (BONA-AL $10 \mathrm{~mm}$ diameter, $5 \mathrm{~cm}$ total length; Standard Sci Tech 
Inc., Seoul, Korea) with a 7-Fr nasocystic drainage tube was inserted for additional lavage and aspiration (Fig. 2D, E). The patient inadvertently removed the nasocystic tube 2 days after the procedure, but the FCSEMS was found to be well positioned on the X-ray. The clinical hallmarks improved, and the patient was discharged 12 days later. An abdominal computed tomography scan 4 weeks later showed complete resolution of the ruptured pseudocyst and perisplenic fluid collection (Fig. $3 \mathrm{~A}, \mathrm{~B})$. The metal stent that was placed for gastrocystostomy was removed. The patient recovered without any recurrent symptoms.
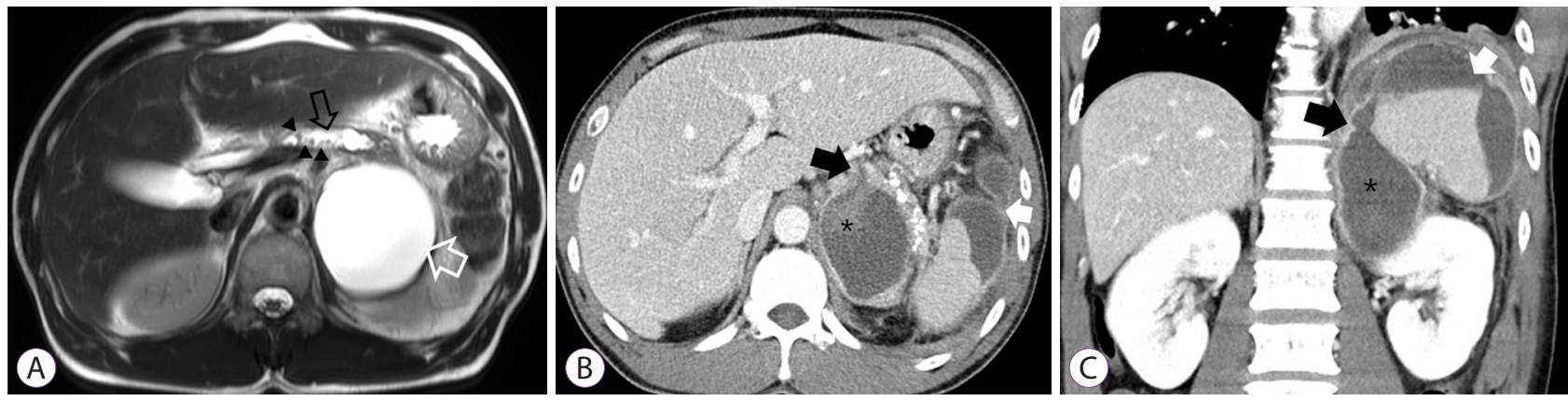

Fig. 1. (A) Axial T2-weighted magnetic resonance imaging showing the main pancreatic duct dilation (open black arrow) with multiple pancreatoliths (black arrowheads) and a pseudocyst (open white arrow), about $9 \mathrm{~cm}$ in size, in the pancreatic tail. Abdominal computed tomography scan of a hemorrhagic pancreatic pseudocyst that ruptured spontaneously. (B) Axial and (C) coronal views showing mural discontinuity (black arrows) of the hemorrhagic pancreatic pseudocyst (asterisks), extension of fluid into the perisplenic space (white arrows), and multiple main pancreatic duct stones with pancreatic atrophy.
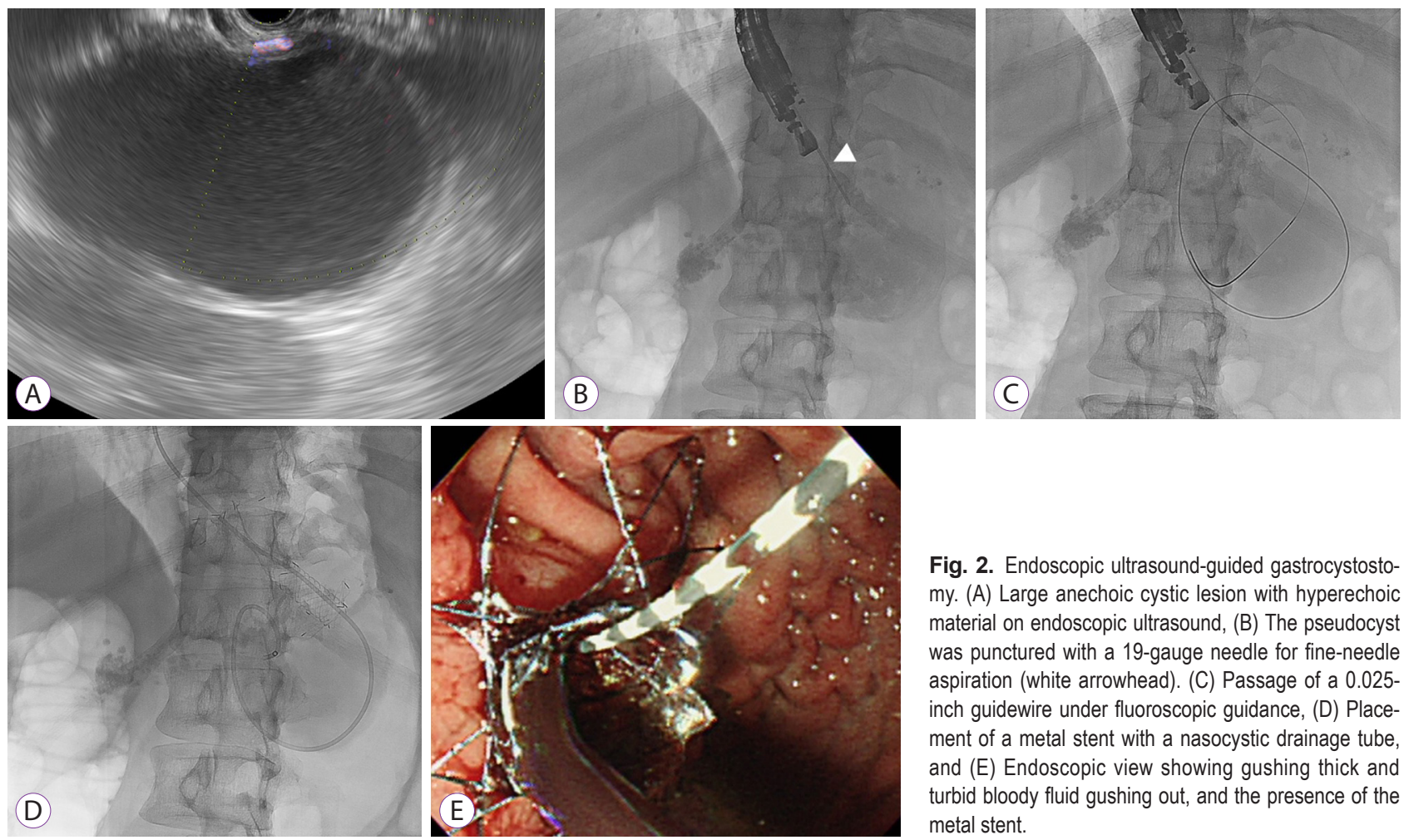

Fig. 2. Endoscopic ultrasound-guided gastrocystostomy. (A) Large anechoic cystic lesion with hyperechoic material on endoscopic ultrasound, (B) The pseudocyst was punctured with a 19-gauge needle for fine-needle aspiration (white arrowhead). (C) Passage of a 0.025inch guidewire under fluoroscopic guidance, (D) Placement of a metal stent with a nasocystic drainage tube, and $(E)$ Endoscopic view showing gushing thick and turbid bloody fluid gushing out, and the presence of the metal stent. 

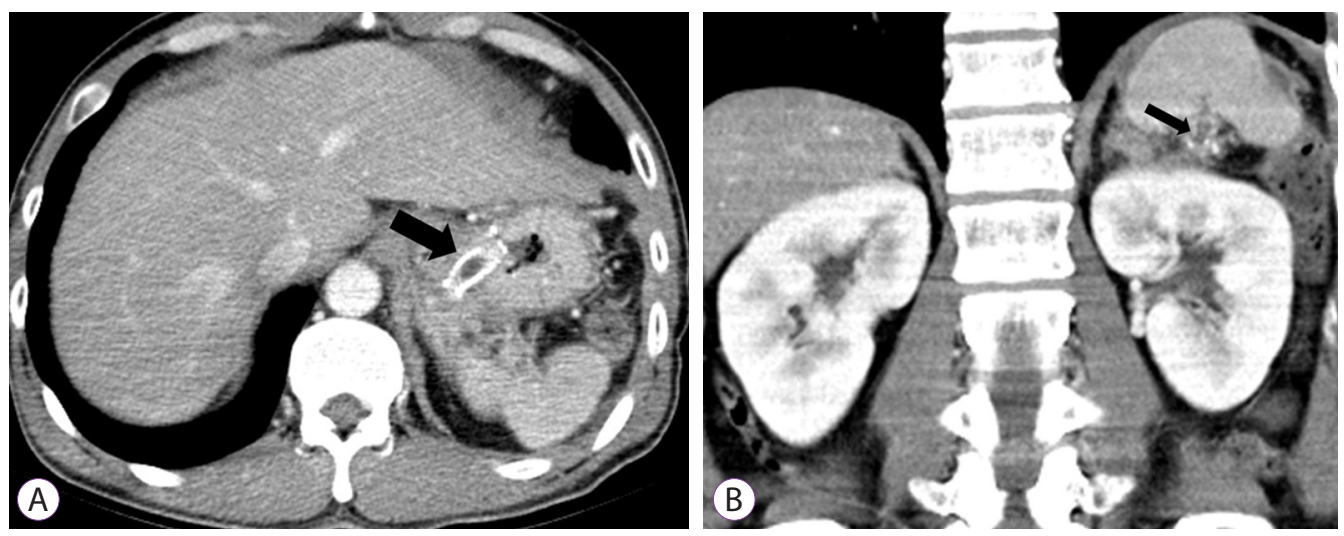

Fig. 3. Abdominal computed tomography scan showing complete resolution of the ruptured pancreatic pseudocyst with the metal stent (black arrow) on axial (A) and coronal (B) views.

\section{DISCUSSION}

Spontaneous rupture of a hemorrhagic pancreatic pseudocyst is rare; however, it can be fatal if not properly managed. The exact mechanism is still unknown. One possible explanation of its occurrence in the current case could be that the pathologic changes, such as its erosion or disruption due to either severe inflammation or the activated lytic enzymes in the pseudocyst, in a superficial vessel may have weakened the pseudocyst wall, subsequently resulting in the spontaneous rupture of the hemorrhagic pseudocyst. ${ }^{3}$ Pseudocyst rupture into the gastrointestinal tract may lead to bleeding or spontaneous regression; however, rupture into the peritoneal cavity may result in peritonitis or shock that requires urgent surgical intervention. ${ }^{4,5}$ The presence of a high concentration of enzymes, such as amylase, lipase, and other proteolytic enzymes, in the pseudocyst can lead to its invasion into adjacent structures. ${ }^{6}$ Traditional management of a ruptured pancreatic pseudocyst involves surgical treatment such as Roux-en-Y cystojejunostomy, distal pancreatectomy, and lavage. ${ }^{7}$

EUS-guided drainage has been reported to be a valuable treatment option for pancreatic pseudocysts. ${ }^{8}$ However, its usefulness in treating ruptured pancreatic pseudocysts is limited. In the present case, hemorrhagic cystic fluid from the ruptured pancreatic pseudocyst only occupied the area between the pancreatic tail and the perisplenic space because of the splenorenal and the gastrosplenic ligaments. Therefore, EUS-guided drainage was considered as the primary treatment, and complete resolution of the ruptured pseudocyst was achieved after the procedure. Substantial controversies remain regarding the use of plastic stents or FCSEMS during EUS-guided pseudocyst drainage. FCSEMSs conserve more time, are technically easier to use, have a higher treatment success rate, and require a shorter period for pseudocyst resolution than plastic stents. ${ }^{9}$ In particular, in pseudocysts with thick debris, as in our patient, an FCSEMS may be a more suitable option with EUS-guided drainage.

In conclusion, according to our experience, EUS-guided drainage with an FCSEMS may be considered an effective alternative to surgery in cases where there is a localized fluid collection due to the ruptured pancreatic pseudocyst.

Conflicts of Interest

The authors have no potential conflicts of interest.

Funding

This paper was supported by "Wonkwang University 2021".

Author Contributions

Conceptualization: Hyung Ku Chon

Data curation: HKC

Formal analysis: Tae Hyeon Kim, HKC

Funding acquisition: HKC

Resources: Chan Park, HKC

Supervision: HKC

Validation: HKC

Writing-original draft: CP, HKC

Writing-review\&editing: CP, THK

ORCID

Chan Park:

Tae Hyeon Kim:

Hyung Ku Chon:

https://orcid.org/0000-0002-2358-7380 https://orcid.org/0000-0002-9723-2136 https://orcid.org/0000-0002-6068-3849

\section{REFERENCES}

1. Song TJ, Lee SS. Endoscopic drainage of pseudocysts. Clin Endosc 2014;47:222-226. 
2. Habashi S, Draganov PV. Pancreatic pseudocyst. World J Gastroenterol 2009;15:38-47.

3. Urakami A, Tsunoda T, Kubozoe T, Takeo T, Yamashita K, Imai H. Rupture of a bleeding pancreatic pseudocyst into the stomach. J Hepatobiliary Pancreat Surg 2002;9:383-385.

4. Hiraishi H, Terano A. Images in clinical medicine. Rupture of a pancreatic pseudocyst into the duodenum. N Engl J Med 1999;340:1411.

5. Rocha R, Marinho R, Gomes A, et al. Spontaneous rupture of pancreatic pseudocyst: report of two cases. Case Rep Surg 2016;2016:7056567.

6. Patidar Y, Sureka B, Singh VP, Bansal K, Maiwall R. Spontaneous rup- ture of intrahepatic pseudocyst into the inferior vena cava. Gastroenterol Rep (Oxf) 2018;6:225-227.

7. Mujer MT, Rai MP, Atti V, Shrotriya S. Spontaneous rupture of a pancreatic pseudocyst. BMJ Case Rep 2018;2018:bcr2018226296.

8. Alali A, Mosko J, May G, Teshima C. Endoscopic ultrasound-guided management of pancreatic fluid collections: update and review of the literature. Clin Endosc 2017;50:117-125.

9. Bang JY, Varadarajulu S. Metal versus plastic stent for transmural drainage of pancreatic fluid collections. Clin Endosc 2013;46:500-502. 